\title{
Ultra-compact X-ray binaries with high luminosity: a key for a new scenario
}

\author{
Konstantin Pavlovskii and Natalia Ivanova \\ Dept. of Physics, University of Alberta, \\ 11322-89 Ave, Edmonton, AB, T6G 2E7, Canada \\ email: pavlovsk@ualberta.ca, nata.ivanova@ualberta.ca
}

\begin{abstract}
Ultra-compact X-ray binaries (UCXBs) are accreting systems with periods less than 1 hour, which qualifies them to contain a degenerate donor-companion. One would expect such systems to have the easiest theoretical explanation, compared to other kinds of X-ray binaries. Nonetheless, current theory fails to explain high mass transfer (MT) rates in three recently well observed long-period UCXBs. We find that this range of MT rates can be maintained if the donor is a remnant of an out-of-thermal-equilibrium naked core of a giant which was revealed in a very recent episode of a common envelope $(\mathrm{CE})$ event.
\end{abstract}

Keywords. accretion, accretion disks, binaries: close, X-rays: binaries

\section{Too high MT rates in UCXBs}

Recent observations show that, at long orbital periods $\geqslant 40$ minutes, there are two groups of UCXBs widely separated in their MT rates (Heinke et al. 2012). The first group is well known and consists of transient sources with very low average MT rates, $\sim 10^{-11} M_{\odot}$ per yr. However, unexpectedly, the other group consists of permanent sources with average MT rates of at least two order of magnitude higher for the same orbital periods (Fig. 1). While in the first group an UCXB can belong to either the Galactic field, or be located in the direction of the bulge or in a globular cluster, in the second group all three UCXBs - 4U 1626-67, 4U 0614+09, 4U 1916-053 - belong only to the Galactic field.

The first group of long-period transient UCXBs is rather well understood in terms of MT sequence of cooling white dwarfs (WDs). The consideration that WDs are not completely degenerate at the start of the MT, and hence have some final entropy in the center, provides a range in possible MT rates for the same period. This effect is minimal for large periods, as MT was going on for already very long time and WDs are close to completion of their cooling (Deloye and Bildsten 2011, see also Fig. 1).

As for the second group of long-period persistent UCXBs, gravitational wave radiation can not provide such high MT rate even if a WD donor has finite entropy. Tidal torque provided by a circumbinary disk (CBD) can provide a stronger angular momentum loss leading to higher MT rates. However, to explain the observed MT rates (as shown on Fig. 1), the fraction of mass ending in CBD has to exceed by 10 times the one predicted by the model calibrated on cataclysmic variables (Shao and Li 2012). Other previously considered donors - an initially slightly evolved main sequence donor with a He-rich core (Nelson et al. 1986, Podsiadlowski et al. 2002), or a naked He star produced by a CE event (Yungelson 2008) - will produce MT rates too low to match observations: at long orbital periods these donors were found to be almost fully degenerate. 


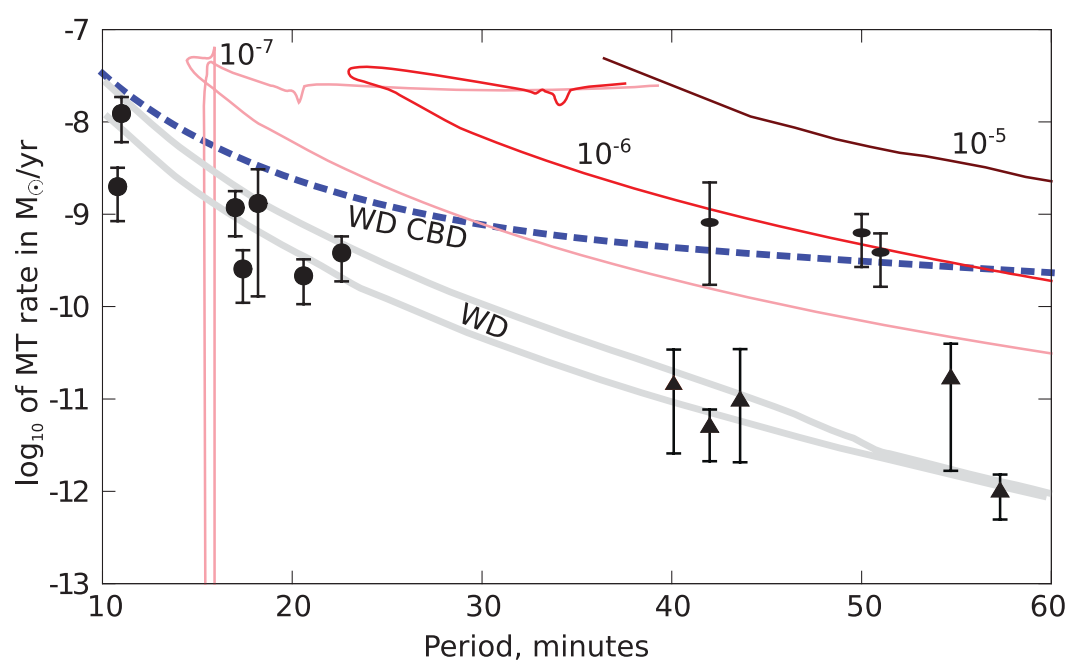

Figure 1. Periods and MT rates for observed UCXBs compared to theoretical MT tracks of: a) partially degenerate WD donors (thick solid lines); b) WD donors evolved with a tidal torque of CBD where the mass ending up in CBD is adopted to be 10 times higher than in a calibrated model (thick dashed line); c) He remnants evolved under gravitational wave radiation after their formation with fast initial post-CE mass loss rate (thin lines, mass loss rates are indicated on the diagram in units of $\mathrm{M}_{\odot} / \mathrm{yr}$ ). Observational data for persistent sources are shown with circles and ellipses (ellipses denote sources with anomalously high MT rates) and for transient sources with triangles (Heinke et al. 2012). He remnants were obtained using the stellar evolution code MESA (Paxton et al. 2011).

\section{Alternative donors}

A naked He core that is formed in a CE event can be larger than a WD of the same mass. It is crucial that if prior to the CE giant's core was non-degenerate, then during and immediately after the envelope ejection it experiences thermal readjustment. This leads to a fast MT onto a companion, with MT rates reaching $10^{-2} M_{\odot} \mathrm{yr}^{-1}$ at a peak. After a peak, MT rates are smaller but still sufficient to keep the remnant out of thermal equilibrium for a while (Ivanova 2011). How long this fast mass loss goes and how much of the core is removed during this mass loss, is not well established.

Hence, to understand high-MT UCXBs, we need to consider self-consistent He remnants. While previous studies considered evolution of initially homogeneous He stars, we formed them via simplified CE event by evolving a $5 M_{\odot}$ star and stripping its hydrogen rich envelope. We considered cases before the start of the He core burning prior a CE, and after. The formed He remnants were evolved with different fast mass loss rates. The MT rates as of UCXBs were then obtained assuming that these out-of-thermal-equilibrium remnants are placed in a binary with a neutron star companion to start the MT, and the binary evolution is driven by gravitational wave radiation only (see Fig. 1). The remnants formed with an initially higher mass loss rate drive a higher MT rate under gravitational wave radiation because they are further out of their thermal equilibrium - for the same remnant mass, they are more inflated and colder (see Figs. 2 and 3).

We also find that the observed variations in He abundances in accretion disks of these mysterious fast-MT UCXBS do not necessarily require a WD donor. They can be explained by different durations of He core burning that took place in donor before or after the start of the MT. Depending on the post-CE orbital separation and on the mass and entropy of a He remnant, MT can start when the donor either: 


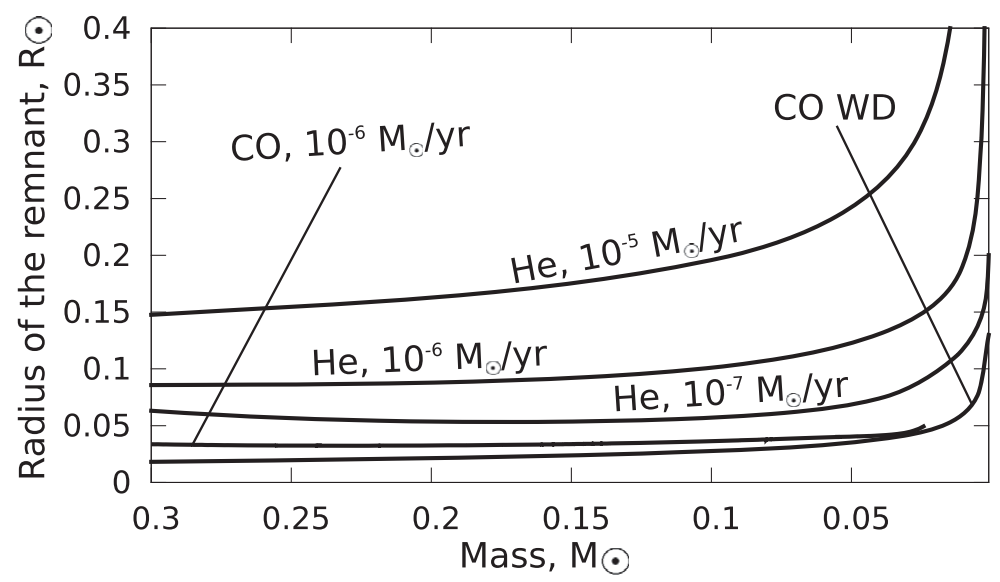

Figure 2. Radii of He and CO remnants evolved with different mass loss rates.

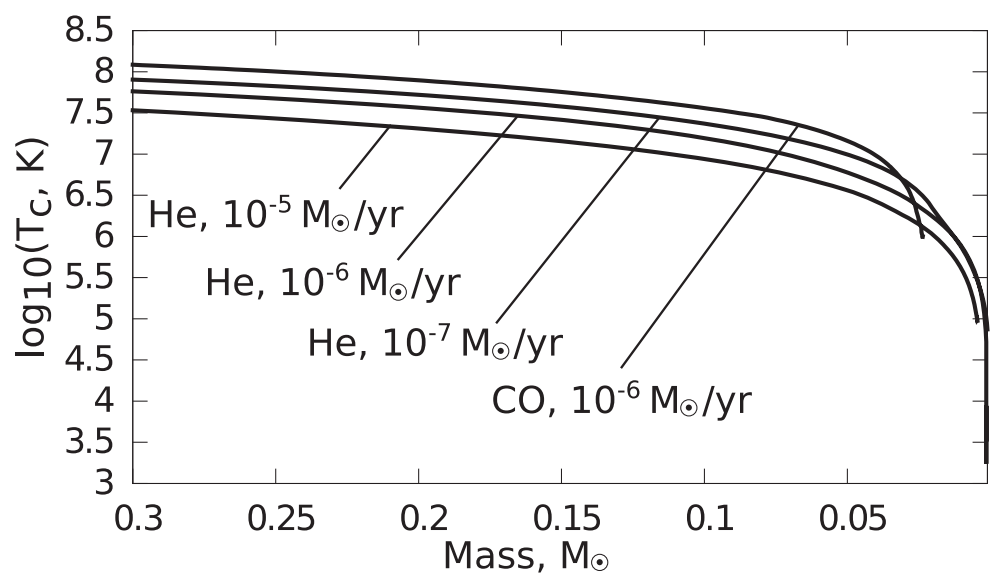

Figure 3. Central temperatures of $\mathrm{He}$ and $\mathrm{CO}$ remnants evolved with different mass loss rate.

- has not yet started He burning - this can be applicable to $4 \mathrm{U} 1916-053$, where the observed accretion disk is nitrogen-rich;

- is going through He burning - in this case the disk will be $\mathrm{C} / \mathrm{O}$ rich, as observed for $4 \mathrm{U} 1626-67$ and $4 \mathrm{U} 0614+09$;

- has already completed He burning. In this case we find that the donor is less likely to be inflated enough to provide the observed MT rates.

\section{References}

Deloye, C. J. \& Bildsten, L. 2011, ApJ, 598, 2, 1217-1228

Heinke, C. O., Engel, M. C., Ivanova, N., Pavlovskii, K., et al. 2012 ApJ submitted, Ivanova, N. 2011, ApJ, 730, 76

Nelson, L. A., Rappaport, S. A., \& Joss, P. C. 1986, ApJ, 304, 231

Paxton, B., Bildsten, L., Dotter, A., Herwig, F., Lesaffre, P., \& Timmes, F. 2011, ApJS, 192, 3

Podsiadlowski, P., Rappaport, S., \& Pfahl, E. D. 2002, ApJ, 565, 1107

Shao, Y. \& Li, X.-D. 2012, ApJ, 745, 165

Yungelson, L. R. 2008, Astronomy Letters, 34, 620 